\title{
Effect of arenobufagin on human pancreatic carcinoma cells
}

\author{
TIANJIAO WANG ${ }^{1}$, ZHUMEI ZHUANG $^{1}$, PENG ZHANG $^{1}$, YUEYUE WANG ${ }^{1}$, LIN MU $^{2}$, \\ HAIFENG JIN $^{1}$, LEI ZHOU ${ }^{1}$, XIAOCHI MA ${ }^{2}$, RUI LIANG ${ }^{1}$ and YUHUI YUAN ${ }^{1}$ \\ ${ }^{1}$ General Surgery, The Second Affiliated Hospital, Institute of Cancer Stem Cell; \\ ${ }^{2}$ College of Pharmacy, Dalian Medical University, Dalian, Liaoning 116044, P.R. China
}

Received June 28, 2015; Accepted June 15, 2017

DOI: $10.3892 / 01.2017 .6798$

\begin{abstract}
Pancreatic carcinoma (PC) is a deadly form of cancer with poor overall survival. Currently, chemotherapy such as gemcitabine and 5-fluorouracil (5-FU) are the most popular medications that can improve survival, but rapid drug-resistance makes the search for more effective drugs urgent. Upon looking for natural components to treat PC, it was found that arenobufagin, a cardiac glycosides-like compound, showed significant effects on the gemcitabine-resistant pancreatic carcinoma cell line Panc-1 and the gemcitabine-sensitive cell line ASPC-1 at nanomolar concentrations. The present study used MTT and clonogenic survival assays to examine survival and proliferation, and western blotting to assess changes in the associated mitogen activated protein kinase and phosphoinositide 3-kinase pathways and expression of apoptosis-related proteins. The current study also detected the cell cycle by flow cytometry. Arenobufagin inhibited cell survival and proliferation, decreased the phosphorylation of key downstream proteins of K-Ras, including protein kinase $\mathrm{B}$ and extracellular signal related kinase, induced cell cycle G2/M phase arrest and apoptosis, and downregulated the level of phosphorylated epidermal growth factor receptor. Notably, the present data also showed that arenobufagin can enhance the sensitivity of PC cells to gemcitabine and 5-FU. In conclusion, arenobufagin could enhance the effect of gemcitabine and 5-FU on PC cells by targeting multiple key proteins. Therefore, arenobufagin has potential as anadjuvant therapy for the treatment of PC.
\end{abstract}

Correspondence to: Professor Yuhui Yuan or Professor Rui Liang, General Surgery, The Second Affiliated Hospital, Institute of Cancer Stem Cell, Dalian Medical University, 9th West Section, Lvshun South Road, Lvshunkou, Dalian, Liaoning 116044, P.R. China E-mail: yuhuiyuan@hotmail.com; yuhuiyuan@dlmedu.edu.cn E-mail: 1r9311038@yahoo.com

Abbreviations: 5-FU, 5-fluorouracil; EGFR, epidermal growth factor receptor; PC, pancreatic carcinoma; PDAC, pancreatic ductal adenocarcinoma; PI, propidium iodide; PI3K, phosphatidylinositol 3-kinase; TCM, traditional Chinese medicine

Key words: pancreatic carcinoma, arenobufagin, epidermal growth factor receptor, drug-resistance

\section{Introduction}

Pancreatic cancer (PC) ranks as the fourth leading cause of cancer-associated mortality in the USA, with the worst prognosis of all solid tumors. It is estimated that 40,560/48,960 patients diagnosed with PC in the USA will succumb to this disease in 2015 (1). Despite advancements in the understanding of the genetics of PC and application of combined chemotherapy and targeted biological agents, the management of this lethal malignancy remains one of the greatest oncological challenges (2). Currently, the only successful treatment for a local pancreatic tumor is surgery, and adjuvant chemotherapy after surgery is indicated to delay relapse, but the effect is limited (3). For those who have advanced and metastatic PC, chemotherapy is a primary treatment and gemcitabine has become the most popular first-line therapy for the treatment of pancreatic ductal adenocarcinoma (PDAC) $(4,5)$. However, the response ratio of PDAC to gemcitabine in clinical research is $<25 \%$, and those patients showing initial response generally develop drug resistance during therapy $(6,7)$. The development of rapid resistance to gemcitabine may be due to either stem-like subpopulations of tumor cells, which have innate resistance to chemotherapy, or be caused by molecular changes in cancer cells, such as alternations of transport and metabolism of gemcitabine or the upregulation of phosphatidylinositol 3-kinase (PI3K)/protein kinase B (Akt) or the DNA repair pathway (8-12). In addition to monotherapy, there are clinical treatments with gemcitabine in combination with other biological or chemotherapeutical targeted agents, such as the epidermal growth factor receptor (EGFR) inhibitors erlotinib, tipifarnib and gefitinib $(13,14)$, but the combinations are limited, with unsatisfactory efficacy. Only patients developing a rapid response upon erlotinib could benefit from EGFR-targeted therapy $(13,15)$. Overall, there is an urgent requirement to identify novel chemotherapeutical agents or an effective combination scheme for this malignancy.

Arenobufagin is a cardiac glycoside agent and one of the main active ingredients of toad secretions. Secretions of the postauricular and skin glands of Bufo gargarizans and Duttaphrynus melanostictus have been used as a traditional Chinese medicine (TCM) in numerous diseases, including cancers, heart failure and sore throat (16). A previous study suggested cardiac glycosides as potent inhibitors of cancer cell growth (17). Previous studies have demonstrated that arenobufagin is a potent $\mathrm{Na}^{+}-\mathrm{K}^{+}$pump inhibitor that depresses the delayed rectifier $\mathrm{K}^{+}$current in myocytes $(18,19)$. It has been 
reported that arenobufagin can suppress cell adhesion, migration and invasion and induce apoptosis and autophagy via inhibition of the PI3K/Akt/mammalian target of rapamycin pathway in a human hepatoma cell line $(20,21)$ as well as block vascular endothelial growth factor (VEGF)-mediated angiogenesis to prevent carcinogenesis (22). However, to the best of our knowledge, the effects and the mechanism of arenobufagin in PC cells have not been studied.

To uncover the effect of arenobufagin on PC cells and the assistance to first-line medicine, the gemcitabine-resistant pancreatic carcinoma cell line Panc-1 and the gemcitabine-sensitive cell line ASPC-1 were used in the present study.

In the current study, it was found that arenobufagin effectively suppressed the proliferation of PC cells by blocking the phosphorylation of both Akt and extracellular signal-regulated kinases (Erk), as well as inducing G2/M phase cell cycle arrest and apoptosis in PC cells. In order to find a new strategy for combination therapy, the present study determined the effect of arenobufagin in combination with gemcitabine or 5-fluorouracil (5-FU), revealing a significant impact on cell proliferation. These results indicate that arenobufagin may be used as a potential adjuvant to overcome the resistance to chemotherapy in PC.

\section{Materials and methods}

Materials. Arenobufagin was isolated from toad secretions, as previously described (23). Gemcitabine and 5-FU were purchased from Sigma-Aldrich (Merck KGaA, Darmstadt, Germany). All 3 agents were dissolved in dimethyl sulfoxide (DMSO) as a stock solution $(10 \mathrm{mM})$ and stored at $-20^{\circ} \mathrm{C}$. The culture media containing different concentrations of these agents were freshly prepared for each experiment. The final concentration of DMSO was $<0.1 \%$. Propidium iodide (PI) for cell cycle analysis was purchased from Sigma-Aldrich (Merck KGaA). Rabbit monoclonal antibodies against Akt (\#4691), phosphorylated Akt $\mathrm{Arr}^{\mathrm{Se}} 73$ (p-Akt) (\#4060), Erk1/2 (\#4695), phosphorylated Erk1/2 (p-Erk; \#4370), EGFR (\#4267), phosphorylated epidermal growth factor receptor ${ }^{\text {Tyr1068 }}$ (p-EGFR; \#3777), caspase-3 (\#9665), poly (ADP-ribose) polymerase (PARP; \#9542), cleaved PARP (Asp214; \#5625), caspase-9 (\#9508) and cleaved caspase-9 (Asp330; \#7237), mouse monoclonal GAPDH (\#97166) and $\beta$-actin (\#3700), and anti-mouse (\#7076) and anti-rabbit (\#7074) IgG-horseradish peroxidase (HRP)-linked antibodies were purchased from Cell Signaling Technology, Inc. (Danvers, MA, USA).

Cell lines and cell culture. The human PC Panc-1 and ASPC-1 cell lines were obtained from the American Type Culture Collection (Manassas, VA, USA). These two cell lines were incubated in Dulbecco's modified Eagle's medium (DMEM; Gibco, Thermo Fisher Scientific, Inc., Waltham, MA, USA) supplemented with $10 \%$ fetal bovine serum (FBS) and $1 \%(\mathrm{v} / \mathrm{v})$ penicillin-streptomycin at $37^{\circ} \mathrm{C}$ under $5 \% \mathrm{CO}_{2}$.

MTT assay. The viability of the Panc-1 and ASPC-1 cells was detected using MTT assay. Cells were planted on 96-well plates with 3 replicates at a density of $5 \times 10^{3}$ cells per well. After culturing in DMEM medium containing 10\% FBS for
$12 \mathrm{~h}$ to obtain a confluent monolayer, the medium was replaced with DMEM containing 5\% FBS and arenobufagin at different concentrations $(0,1,10$ and $100 \mathrm{nM})$ for 24,48 and $96 \mathrm{~h}$.

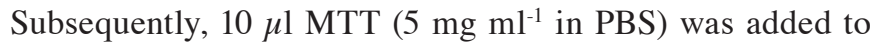
each well at the indicated time points. The culture medium was removed and MTT formazan was dissolved in $150 \mu \mathrm{l}$ DMSO per well $4 \mathrm{~h}$ later. The plates were agitated for $15 \mathrm{~min}$, and $\mathrm{OD}_{490 \mathrm{~nm}}$ was measured using an absorbance reader.

Clonogenic survival assay. Cell proliferation ability was measured using a colony formation assay. Approximately 500 cells were seeded in each well on a 12 -well plate as a single cell suspension. After $24 \mathrm{~h}$, different concentrations of arenobufagin $(0,5,10$ and $50 \mathrm{nM})$ were added and the cells continued to be maintained at $37^{\circ} \mathrm{C}$ with a humidified atmosphere of $5 \% \mathrm{CO}_{2}$ for 15-20 days. Visible colonies were then stained with crystal violet and manually counted.

Western blot analysis. Cells were treated with arenobufagin $(0,5$ and $10 \mathrm{nM})$ at the indicated time $(0,0.5$ and $1 \mathrm{~h})$. Whole-cell extracts were prepared using radioimmunoprecipitation assay buffer supplemented with proteinase inhibitors (Sigma-Aldrich, Merck KGaA) at $4^{\circ} \mathrm{C}$. Following centrifugation at $13,000 \mathrm{x} \mathrm{g}$ for $20 \mathrm{~min}$, the supernatant was collected and quantified using the bicinchoninic acid protein assay. Total protein (50 $\mu \mathrm{g}$ per well) was separated using 8-12\% SDS-PAGE and transferred to nitrocellulose transfer membranes. The membranes were blocked in TBST (10 mM Tris-HCl, $\mathrm{pH} 7.4$; $150 \mathrm{mM} \mathrm{NaCl} ; 0.1 \%$ Tween-20) with 5\% non-fat milk for $2 \mathrm{~h}$. The membranes were then incubated with specific primary antibodies (all antibodies were diluted at a ratio of 1:1,000) overnight at $4^{\circ} \mathrm{C}$, followed by treatment with HRP-linked secondary antibodies (24). The protein bands were visualized using ECL agent (Thermo Fisher Scientific, Inc.) and detected using a Gel imaging system (Bio-Rad Laboratories, Inc., Hercules, California, USA).

Detection of cell cycle. Cells were seeded on a 6-well plate and treated with $100 \mathrm{nM}$ arenobufagin for $24 \mathrm{~h}$. The cells were then harvested by trypsin (up to $5 \times 10^{6}$ cells), washed twice with PBS, and fixed with cold $70 \%$ ethanol at $4^{\circ} \mathrm{C}$ overnight. The cells were stained with $50 \mu \mathrm{g} / \mathrm{ml}$ PI [PBS $480 \mu \mathrm{l} ; 5 \mu \mathrm{l}$ PI (5 mg/ml); $5 \mu \mathrm{l}$ RNase (10 mg/ml); $10 \mu \mathrm{l}$ Triton-100 (10\%)] at $37^{\circ} \mathrm{C}$ for $30 \mathrm{~min}$ and were kept in the dark. The cells were then suspended in PBS and cell cycle distribution was detected. Samples were analyzed using a FACSC flow cytometer. Additional analyses were processed by Flow Jo software (Tree Star, Inc., Ashland, OR, USA) (24).

Statistical analysis. The data were analyzed by Student's t-test or one-way analysis of variance (Dunnett's test and Least Significant Difference test). $\mathrm{P}<0.05$ was considered to indicate a statistically significant difference. All analysis was performed using SPSS13.0 (SPSS, Inc., Chicago, IL, USA).

\section{Results}

Arenobufagin inhibited survival and proliferation of PC cells. The chemical structure of arenobufagin is shown in Fig. 1A. The cytotoxic efficacy of arenobufagin was first tested on the 


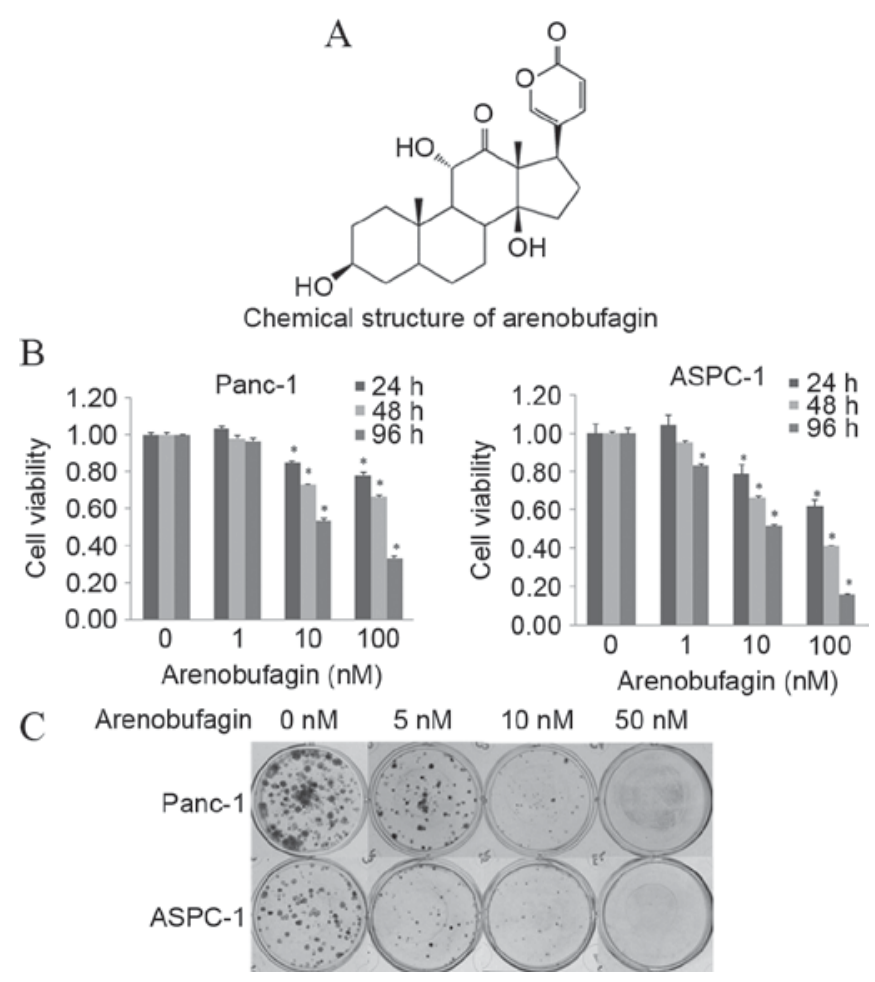

Figure 1. Arenobufagin inhibited cell survival and proliferation in human PC cells. (A) The chemical structure of arenobufagin. (B) Cell viability was measured by MTT. Panc-1 and ASPC-1 cell lines were treated with different concentrations of arenobufagin $(0-100 \mathrm{nM})$ for 24,48 and $96 \mathrm{~h}$, and cell viability was evaluated by optical density at $490 \mathrm{nM}$. The results showed that arenobufagin inhibits the survival of PC cells significantly at concentrations as low as $10 \mathrm{nM}$. (C) Cell proliferation was tested using a clonogenic survival assay. Each cell line was treated with arenobufagin at 0,5,10 and $50 \mathrm{nM}$. As shown, the proliferation of PC cells was evidently inhibited by arenobufagin, even at $5 \mathrm{nM}$. The data shown are representative of at least three experiments. ${ }^{\text {" }} \mathrm{P}<0.05$ compared with the control; cells treated with $0 \mathrm{nM}$ were used as the control. PC, pancreatic cancer.

PC Panc-1 and ASPC-1 cell lines by MTT analysis. Subsequent to treatment with different concentrations $(0-100 \mathrm{nM})$ of arenobufagin for 24, 48 and $96 \mathrm{~h}$, cell proliferation was significantly suppressed in a dose- and time-dependent manner, as shown in Fig. 1B. To confirm the inhibition of cellular proliferation, the present study processed the colony formation assay (Fig. 1C). The MTT assay and the colony formation assay showed that cellular viability and proliferation could be inhibited by arenobufagin even at a concentration as low as $1 \mathrm{nM}$.

Arenobufagin suppressed the phosphorylation of Akt, Erk and EGFR. The present study investigated whether arenobufagin could inhibit key downstream pathways of cell survival and proliferation, and whether they play a role in arenobufagin-mediated cell death. As shown in Fig. 2A, western blot analysis showed that the phosphorylation of Akt and Erk decreased in ASPC-1 and Panc-1 cell lines within $1 \mathrm{~h}$ when treated with arenobufagin at $10 \mathrm{nM}$. To uncover the mechanism of arenobufagin on PC cells, the present study examined the state of EGFR. Subsequent to treatment with arenobufagin at $10 \mathrm{nM}$ for 0 and $0.5 \mathrm{~h}$, the levels of p-EGFR decreased in the Panc-1 cell line. There was no evident change in the gemcitabine-sensitive ASPC-1 cells, but it should be noted that the initial level of p-EGFR was low in the ASPC-1 cell line, as shown in Fig. 2B.
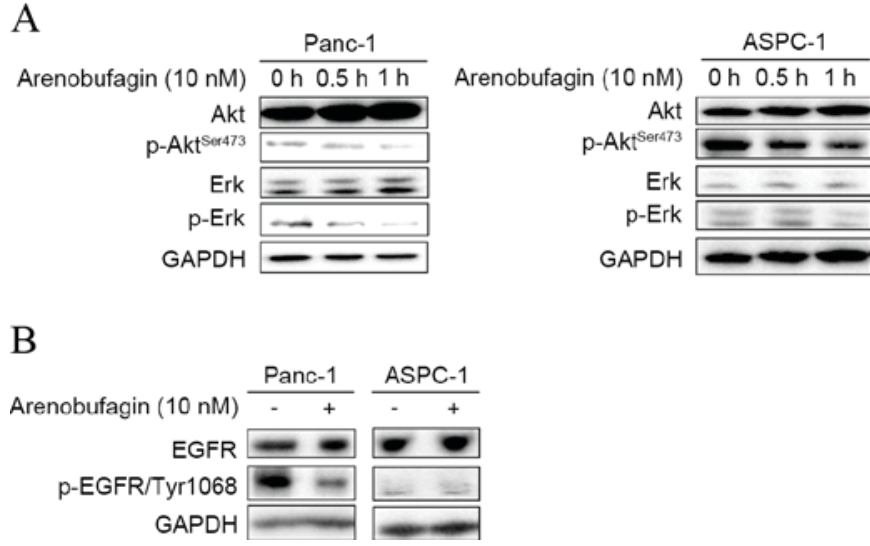

Figure 2. Arenobufagin decreased the phosphorylation of Akt, Erk and EGFR. (A) Levels of p-Erk and p-Akt ${ }^{\mathrm{Ser} 437}$ in total cell lysates from Panc-1 and ASPC-1 cells treated with arenobufagin at $10 \mathrm{nM}$ for 0.5 and $1 \mathrm{~h}$ were decreased compared with the control, which was untreated. (B) Cells were treated as aforementioned. The levels of total EGFR and phosphorylated EGFR were detected and the phosphorylated proteins were decreased. GAPDH was used as a loading control, and the ratios were compared to the GAPDH at $0 \mathrm{~h}$. The western blots shown here are representative of at least three independent experiments. p-, phosphorylated; Erk, extracellular signal-regulated kinases; EGFR, epidermal growth factor receptor.

Arenobufagin induced cell cycle G2/M phase arrest and G0/S phase decline. The inhibition of cellular proliferation is usually caused by cell cycle arrest and cell death. Given the significant decrease in cell viability, a dose of $10 \mathrm{nM}$ of arenobufagin was used to explore the underlying mechanism of arenobufagin-induced inhibition on cellular proliferation. Subsequent to treatment with the indicated concentration for $24 \mathrm{~h}$, the percentage of cells in the $\mathrm{G} 2 / \mathrm{M}$ phase increased from $10.3-22.74 \%$ in Panc-1 cells and 16.46-30.65\% in ASPC-1 cells, along with a decrease in the $\mathrm{S}$ phase, as shown in Fig. $3 \mathrm{~A}$ and $\mathrm{B}$. The statistical analysis also showed that the increase in the $\mathrm{G} 2 / \mathrm{M}$ phase percentage was significant $(\mathrm{P}<0.05)$.

Arenobufagin affected the apoptosis-related pathway. In addition to cell cycle arrest, the effect of arenobufagin on the apoptosis pathway in PC cells was examined. Western blot analysis revealed not only specific cleavage of PARP but also a decrease in PARP, pro-caspase-3 and pro-caspase-9 was induced by arenobufagin treatment (Fig. 4A). The decrease in caspase-9 (Fig. 4B) indicated that the mechanism of apoptosis may be associated with a mitochondrial-dependent pathway.

Arenobufagin significantly enhanced the effects of both gemcitabine and 5-FU. The present study assessed the effect of arenobufagin on the inhibition of proliferation induced by gemcitabine in the gemcitabine-resistantPanc-1 and gemcitabine-sensitive ASPC-1cell lines. The two cell lines were treated with arenobufagin and gemcitabine at the indicated concentrations, either alone or in combination for $48 \mathrm{~h}$. The combination of arenobufagin and gemcitabine inhibited the growth of Panc-1 and ASPC-1 cells more than either of the agentsalone, with the cell viability of Panc-1 cells declining from $89 \%$ in the gemcitabine group and $71 \%$ in the arenobufagin group to $50 \%$ in the combination treatment group. In the ASPC-1 cell line, the viability of cells declined from $71 \%$ in the gemcitabine group and $51 \%$ in the 
A

Panc-1
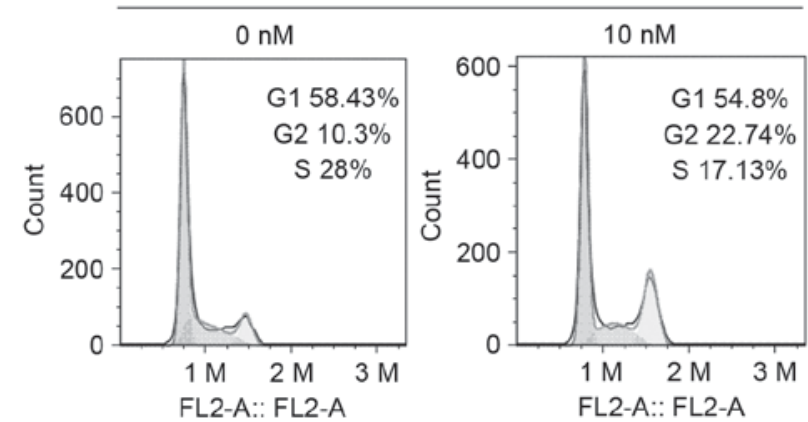

ASPC-1
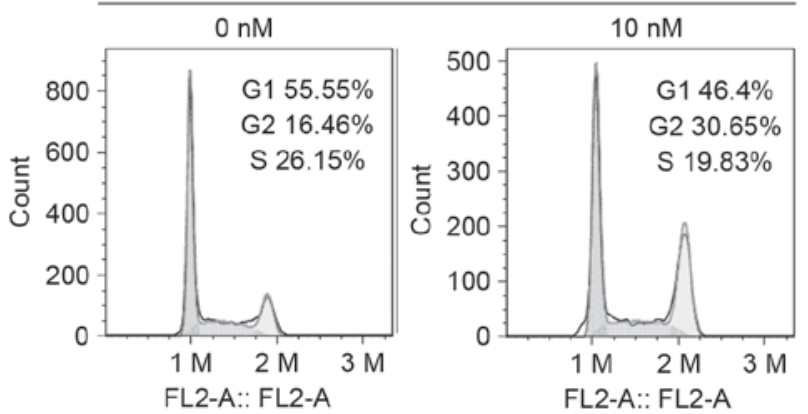

B
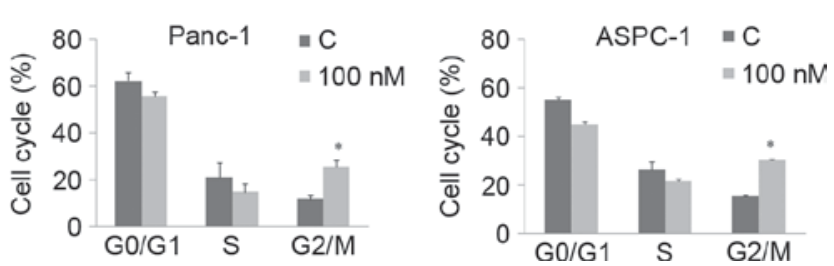

Figure 3. Arenobufagin leads to cell cycle G2/M phase arrest. (A) Cells were exposed to arenobufagin at $10 \mathrm{nM}$ for $24 \mathrm{~h}$ and were collected by trypsinization and analyzed by flow cytometry subsequent to staining with propidium iodide. Arenobufagin induced G2/M phase cell cycle arrest in Panc-1 and ASPC-1 cells. (B) Three independent experiments were performed, the histogram is presented according to statistical analysis. ${ }^{*} \mathrm{P}<0.05$ compared with control; groups treated for $0 \mathrm{~h}$ were used as the control. C, control.

arenobufagin group to $41 \%$ in the combination treatment group. The decline between the single treatment groups and the combination groups was statistically significant $(\mathrm{P}<0.05$; Fig. 5A). In addition, the present study identified that the combination treatment of arenobufagin at $10 \mathrm{nM}$ and 5-FU at $50 \mu \mathrm{M}$ for $48 \mathrm{~h}$ significantly enhanced $(\mathrm{P}<0.05)$ the effect of 5-FU, with the cell viability of Panc-1 cells decreasing between $82 \%$ in the 5-FU group and 50\% in the combination group. The viability of ASPC-1 cells decreased between 68\% in the 5-FU group to $42 \%$ in the combination treatment group (Fig. 5B).

\section{Discussion}

Arenobufagin, an effective cardiac glycoside, is one of the most active compounds found in toad secretions, and is listed in the Chinese Pharmacopoeia (20). Arenobufagin has been used to treat hepatic carcinoma in TCM. Certain cardiac glycosides have been reported as potent inhibitors of cancer cell growth (17). To the best of our knowledge, the present study demonstrated for the first time that arenobufagin is an
A
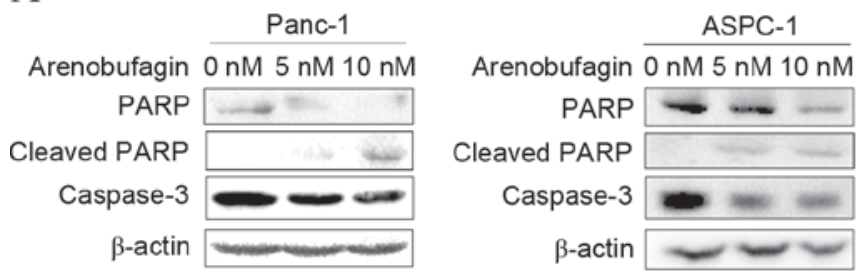

B
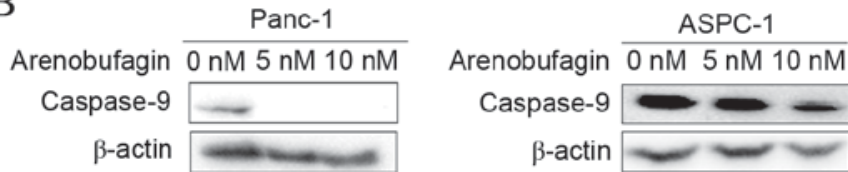

Figure 4. Effects of arenobufagin on apoptosis in prostate cancer cells (A) Panc-1 and Aspc-1 cells were treated with different concentrations of arenobufagin $(0,5$ and $10 \mathrm{nM})$ for $48 \mathrm{~h}$. Western blot analysis demonstrated that the expression of the apoptosis-related proteins PARP and caspase-3 decreased and the expression of cleaved PARP increased. (B) Cells were treated as in (A) and the level of caspase- 9 was detected. The groups treated with arenobufagin also showed decrease in caspase- 9 . $\beta$-actin was used as the internal control. The western blots shown are representative of at least 3 independent experiments with similar results. PARP, poly (ADP-ribose) polymerase.
A

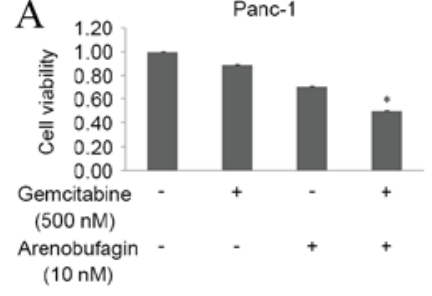

B $1.20 \quad$ Panc-1

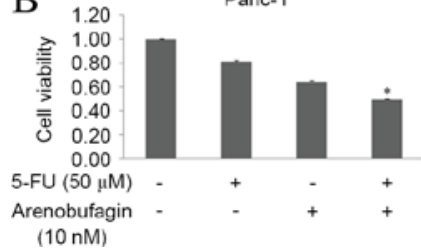

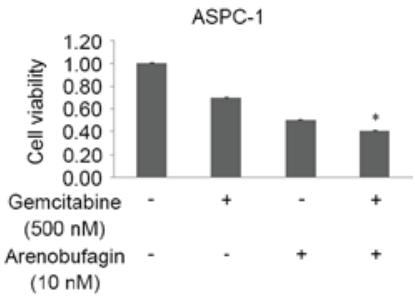

ASPC-1

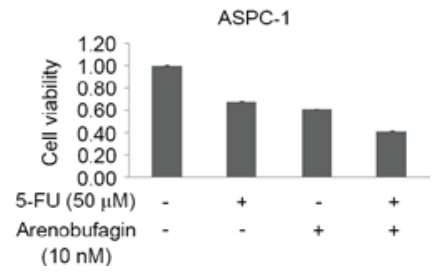

Figure 5. Arenobufagin enhanced the effects of gemcitabine and 5-FU. (A) Panc-1 and ASPC-1 cells were treated with arenobufagin $(10 \mathrm{nM})$ and/or gemcitabine $(500 \mathrm{nM})$ for $48 \mathrm{~h}$. The combination of the two drugs significantly inhibited the proliferation of Panc-1 more than either arenobufagin or gemcitabine alone. (B) Panc-1 and ASPC-1 cells were treated with arenobufagin $(10 \mathrm{nM})$ and/or 5-FU $(50 \mu \mathrm{M})$ for $48 \mathrm{~h}$. The combination of the two drugs inhibited the proliferation of Panc-1 more than either arenobufagin or 5-FU alone. The data shown are representative of at least three independent experiments. ${ }^{*} \mathrm{P}<0.05$ compared with the monotherapy group. 5-FU, poly (ADP-ribose) polymerase.

effective agent in PC cell lines, including the drug-resistant Panc-1cell line.

K-Ras has previously been identified as a small GTPase that is mutated in $90 \%$ of human pancreatic carcinomas (25). Certain genetic studies have shown that K-Ras activation and mutation is necessary for the initiation of PC (26-28), and an inducible pancreas-specific expression system was used to show that K-Ras expression is also required for tumor maintenance (29). These mutations lock K-Ras and its downstream proteins, such as Akt and Erk, in a constitutively activated form. In turn, this leads to enhanced cell proliferation and a 
growth advantage to the cancer cell, as well as playing a key role in tumorigenesis and resistance to standard therapies, such as chemotherapy and radiation $(30,31)$. Thus, the associated signaling pathways are critical targets for which specific inhibitors are expected to exert antitumor efficacy. It is notable that arenobufagin could evidently downregulate the phosphorylation of Erk and Akt in the K-Ras mutant Panc-1 cell line, suggesting that arenobufagin may be an efficient inhibitor in Ras mutation cancer cell lines. In addition, the present study showed that this downregulation was more evident on the level of p-Erk compared with p-Akt when treated with arenobufagin, which is consistent with previous studies (32).

A new study recently showed that EGFR signaling is essential for K-Ras oncogene-driven PDAC (33). EGFR belongs to the tyrosine kinase receptor erbB family, and is important in tumor growth, metastasis and disease recurrence $(34,35)$. Overexpression of EGFR commonly occurs in PC, and this overexpression is associated with a poor outcome (36-38). Although EGFR inhibitors have exerted significant clinical benefits, clinical research has shown that only patients who developed a rapid response upon erlotinib treatment benefit from EGFR inhibitors (13), which suggests a limited efficacy of EGFR inhibition and the requirement for agents targeting multiple signaling pathways. Considering the inhibitory effects of arenobufagin on the phosphorylation of Erk and Akt in K-Ras mutant Panc-1 cell lines and the cross-talk between EGFR and K-Ras, it was hypothesized that a possible target by which arenobufagin may affect cell growth and death was EGFR. The present study detected significant decreases of phosphorylated EGFR subsequent to treatment with arenobufagin in PC cells, thus confirming that arenobufagin is an efficient agent for the treatment of Ras mutated PC.

Previously, it has been reported that arenobufagin induces apoptosis in hepatocellular carcinoma cells. Similar to the present findings for arenobufagin, certain studies have reported that bufalin, another representative cardiac glycoside compound from secreted toad toxins, inhibited cell proliferation in various cancer cells and induced apoptosis and cell cycle arrest in PC cells $(21,39-41)$. The exact mechanism by which arenobufagin induces apoptosis in pancreatic cells is unclear. The present finding on the reduction of caspase- 9 indicated that arenobufagin may induce apoptosis via a mitochondrial pathway in PC cells. In addition, to the best of our knowledge, the present data showed for the first time that arenobufagin induced cell cycle G2/M phase arrest and a decrease in the population of cells in the $\mathrm{S}$ phase, inhibiting the proliferation of PC cells.

Several studies have indicated that chemotherapeutic and biological targeted agents could be used in combination with clinical chemotherapeutic drugs, such as gemcitabine and 5-FU to overcome drug resistance and improve the efficiency of treatments $(42,43)$. The classic model for PDAC treatment is treatment with gemcitabine as a single agent or in combination with EGFR inhibitors. Since it has been reported that the levels of EGFR and Erk phosphorylation in PDAC were increased in Panc-1 and BXPC-1 cell lines when treated with gemcitabine, this may be a mechanism of drug resistance (15). The phosphorylation of Erk is associated with cell proliferation, differentiation, migration and transcription. Notably, the present western blot analysis showed that arenobufagin could significantly downregulate the phosphorylation of EGFR, Erk and Akt. Cell growth analysis testified to the effect of arenobufagin in combination with gemcitabine or 5-FU, and the results demonstrated that the involvement of arenobufagin could significantly enhance the inhibiting effect of either gemcitabine or 5-FU against PC cells. Thus, the present results indicated that arenobufagin could be used as a new drug to enhance the effect of gemcitabine through inhibiting EGFR, Erk, Akt and NF- $\mathrm{NB}$ associated pathways.

In summary, the present study determined that arenobufagin could cause cell cycle G2/M phase arrest and apoptosis in PC cells, and downregulate the levels of p-Erk, p-Akt and p-EGFR even in drug-resistant Panc- 1 cell line, which could in turn offset the adverse effects of gemcitabine. Thus, the present study identified arenobufagin as a potential effective agent with a marked effect on pancreatic cancer cells, and therefore causing us to consider arenobufagin as a promising candidate for combination therapy in PC.

\section{Acknowledgements}

This study was supported by funds from the Liaoning Province Natural Science Foundation of China (grant no. 2013023043) and National Natural Science Foundation of China (grant no. 510575).

\section{References}

1. Siegel RL, Miller KD and Jemal A: Cancer statistics, 2015. CA Cancer J Clin 65: 5-29, 2015.

2. Hidalgo M: Pancreatic cancer. N Engl J Med 362: 1605-1617, 2010.

3. Vincent A, Herman J, Schulick R, Hruban RH and Goggins M: Pancreatic cancer. Lancet 378: 607-620, 2011.

4. Burris HA III, Moore MJ, Andersen J, Green MR, Rothenberg ML, Modiano MR, Cripps MC, Portenoy RK, Storniolo AM, Tarassoff P, et al: Improvements in survival and clinical benefit with gemcitabine as first-line therapy for patients with advanced pancreas cancer: A randomized trial. J Clin Oncol 15: 2403-2413, 1997.

5. Berlin J and Benson AB III: Chemotherapy: Gemcitabine remains the standard of care for pancreatic cancer. Nat Rev Clin Oncol 7: 135-137, 2010.

6. Ducreux M, Boige V and Malka D: Treatment of advanced pancreatic cancer. Semin Oncol 34 (2 Suppl 1): S25-S30, 2007.

7. Ying JE, Zhu LM and Liu BX: Developments in metastatic pancreatic cancer: Is gemcitabine still the standard? World J Gastroenterol 18: 736-745, 2012.

8. Davidson JD, Ma L, Flagella M, Geeganage S, Gelbert LM and Slapak CA: An increase in the expression of ribonucleotide reductase large subunit 1 is associated with gemcitabine resistance in non-small cell lung cancer cell lines. Cancer Res 64: 3761-3766, 2004.

9. Itoi T, Sofuni A, Fukushima N, Itokawa F, Tsuchiya T, Kurihara T, Moriyasu F, Tsuchida A and Kasuya K: Ribonucleotide reductase subunit M2 mRNA expression in pretreatment biopsies obtained from unresectable pancreatic carcinomas. J Gastroenterol 42: 389-394, 2007.

10. Nakano Y, Tanno S, Koizumi K, Nishikawa T, Nakamura K, Minoguchi M, Izawa T, Mizukami Y, Okumura T and Kohgo Y: Gemcitabine chemoresistance and molecular markers associated with gemcitabine transport and metabolism in human pancreatic cancer cells. Br J Cancer 96: 457-463, 2007.

11. Hagmann W, Jesnowski R and Löhr JM: Interdependence of gemcitabine treatment, transporter expression, and resistance in human pancreatic carcinoma cells. Neoplasia 12: 740-747, 2010.

12. Ng SS, Tsao MS, Nicklee T and Hedley DW: Wortmannin inhibits pkb/akt phosphorylation and promotes gemcitabine antitumor activity in orthotopic human pancreatic cancer xenografts in immunodeficient mice. Clin Cancer Res 7: 3269-3275, 2001. 
13. Moore MJ, Goldstein D, Hamm J, Figer A, Hecht JR, Gallinger S, Au HJ, Murawa P, Walde D, Wolff RA, et al: Erlotinib plus gemcitabine compared with gemcitabine alone in patients with advanced pancreatic cancer: A phase III trial of the National Cancer Institute of Canada Clinical Trials Group. J Clin Oncol 25: 1960-1966, 2007.

14. Fountzilas G, Bobos M, Kalogera-Fountzila A, Xiros N, Murray S, Linardou H, Karayannopoulou G, Koutras AK, Bafaloukos D, Samantas E, et al: Gemcitabine combined with gefitinib in patients with inoperable or metastatic pancreatic cancer: A phase II Study of the Hellenic Cooperative Oncology Group with biomarker evaluation. Cancer Invest 26: 784-793, 2008.

15. Venkatasubbarao K, Peterson L, Zhao S, Hill P, Cao L, Zhou Q, Nawrocki ST and Freeman JW: Inhibiting signal transducer and activator of transcription-3 increases response to gemcitabine and delays progression of pancreatic cancer. Mol Cancer 12: 104, 2013.

16. Liang Y, Liu AH, Qin S, Sun JH, Yang M, Li P and Guo DA: Simultaneous determination and pharmacokinetics of five bufadienolides in rat plasma after oral administration of Chansu extract by SPE-HPLC method. J Pharm Biomed Anal 46: 442-448, 2008

17. Wang Y, Lonard DM, Yu Y, Chow DC, Palzkill TG, Wang J, Qi R, Matzuk AJ, Song X, Madoux F, et al: Bufalin is a potent small-molecule inhibitor of the steroid receptor coactivators SRC-3 and SRC-1. Cancer Res 74: 1506-1517, 2014.

18. Cruz Jdos S and Matsuda H: Arenobufagin, a compound in toad venom, blocks $\mathrm{Na}(+)-\mathrm{K}+$ pump current in cardiac myocytes. Eur J Pharmacol 239: 223-226, 1993.

19. Cruz Jdos S and Matsuda H: Depressive effects of arenobufagin on the delayed rectifier K+ current of guinea-pig cardiac myocytes. Eur J Pharmacol 266: 317-325, 1994.

20. Cao HH, Zhang DM, Liu JS, Hou CY, Kurihara H and Ye WC: Inhibitory effect of arenobufagin on the adhesion, invasion and migration of human hepatoma carcinoma cells. Chinese Pharmacol Bull 27: 19-23, 2011.

21. Zhang DM, Liu JS, Deng LJ, Chen MF, Yiu A, Cao HH, Tian HY, Fung KP, Kurihara H, Pan JX and Ye WC: Arenobufagin, a natura bufadienolide from toad venom, induces apoptosis and autophagy in human hepatocellular carcinoma cells through inhibition of PI3K/Akt/mTOR pathway. Carcinogenesis 34: 1331-1342, 2013.

22. Li M, Wu S, Liu Z, Zhang W, Xu J, Wang Y, Liu J, Zhang D, Tian H, Li Y and Ye W: Arenobufagin, a bufadienolide compound from toad venom, inhibits VEGF-mediated angiogenesis through suppression of VEGFR-2 signaling pathway. Biochem Pharmacol 83: 1251-1260, 2012.

23. Li J, Ma X, Li F, Wang J, Chen H, Wang G, Lv X, Sun C and Jia J: Preparative separation and purification of bufadienolides from Chinese traditional medicine of ChanSu using high-speed counter-current chromatography. J Sep Sci 33: 1325-1330, 2010.

24. Yuan Y, Qin L, Liu D, Wu RC, Mussi P, Zhou S, Songyang Z and Xu J: Genetic Screening Reveals an Essential Role of p27kip1 in Restriction of Breast Cancer Progression. Cancer Res 67: 8032-8042, 2007.

25. Thomas RK, Baker AC, Debiasi RM, Winckler W, Laframboise T, Lin WM, Wang M, Feng W, Zander T, MacConaill L, et al High-throughput oncogene mutation profiling in human cancer. Nat Genet 39: 347-351, 2007.

26. Aguirre AJ, Bardeesy N, Sinha M, Lopez L, Tuveson DA, Horner J, Redston MS and DePinho RA: Activated Kras and Ink4a/Arf deficiency cooperate to produce metastatic pancreatic ductal adenocarcinoma. Genes Dev 17: 3112-3126, 2003.

27. Hingorani SR, Petricoin EF, Maitra A, Rajapakse V, King C, Jacobetz MA, Ross S, Conrads TP, Veenstra TD, Hitt BA, et al: Preinvasive and invasive ductal pancreatic cancer and its early detection in the mouse. Cancer Cell 4: 437-450, 2003.

28. Hingorani SR, Wang L, Multani AS, Combs C, Deramaudt TB Hruban RH, Rustgi AK, Chang S and Tuveson DA: Trp53R172H and KrasG12D cooperate to promote chromosomal instability and widely metastatic pancreatic ductal adenocarcinoma in mice. Cancer Cell 7: 469-483, 2005.
29. Collins MA, Bednar F, Zhang Y, Brisset JC, Galbán S, Galbán CJ, Rakshit S, Flannagan KS, Adsay NV and Pasca di Magliano M: Oncogenic Kras is required for both the initiation and maintenance of pancreatic cancer in mice. J Clin Invest 122: 639-653, 2012.

30. Shaw RJ and Cantley LC: Ras, PI(3)K and mTOR signalling controls tumour cell growth. Nature 441: 424-430, 2006.

31. Sweet RW, Yokoyama S, Kamata T, Feramisco JR, Rosenberg M and Gross M: The product of ras is a GTPase and the T24 oncogenic mutant is deficient in this activity. Nature 311: 273-275, 1984.

32. Hofmann I, Weiss A, Elain G, Schwaederle M, Sterker D, Romanet V, Schmelzle T, Lai A, Brachmann SM, Bentires-Alj M, et al: K-RAS mutant pancreatic tumors show higher sensitivity to MEK than to PI3K inhibition in vivo. PLoS One 7: e44146, 2012.

33. Navas C, Hernandez-Porras I, Schuhmacher AJ, Sibilia M, Guerra $\mathrm{C}$ and Barbacid M: EGF receptor signaling is essential for k-ras oncogene-driven pancreatic ductal adenocarcinoma. Cancer Cell 22: 318-330, 2012.

34. Pryczynicz A, Guzińska-Ustymowicz K, Czyzewska J and Kemona A: Expression of epidermal growth factors and apoptosis markers in pancreatic ductal adenocarcinoma. Folia Histochem Cytobiol 47: 667-671, 2009

35. Weiss GA, Rossi MR, Khushalani NI, Lo K, Gibbs JF, Bharthuar A, Cowell JK and Iyer R: Evaluation of phosphatidylinositol-3-kinase catalytic subunit (PIK3CA) and epidermal growth factor receptor (EGFR) gene mutations in pancreaticobiliary adenocarcinoma. J Gastrointest Oncol 4: 20-29, 2013.

36. Barr S, Thomson S, Buck E, Russo S, Petti F, Sujka-Kwok I, Eyzaguirre A, Rosenfeld-Franklin M, Gibson NW, Miglarese M, et al: Bypassing cellular EGF receptor dependence through epithelial-to-mesenchymal-like transitions. Clin Exp Metastasis 25: 685-693, 2008.

37. Miyabayashi K, Ijichi H, Mohri D, Tada M, Yamamoto K, Asaoka Y, Ikenoue T, Tateishi K, Nakai Y, Isayama $\mathrm{H}$, et al: Erlotinib prolongs survival in pancreatic cancer by blocking gemcitabine-induced MAPK signals. Cancer Res 73: 2221-2234, 2013.

38. Walters DM, Lindberg JM, Adair SJ, Newhook TE, Cowan CR, Stokes JB, Borgman CA, Stelow EB, Lowrey BT, Chopivsky ME, et al: Inhibition of the growth of patient-derived pancreatic cancer xenografts with the MEK inhibitor trametinib is augmented by combined treatment with the epidermal growth factor receptor/HER2 inhibitor lapatinib. Neoplasia 15: 143-155, 2013.

39. Zhang L, Nakaya K, Yoshida T and Kuroiwa Y: Induction by bufalin of differentiation of human leukemia cells HL60, U937, and ML1 toward macrophage/monocyte-like cells and its potent synergistic effect on the differentiation of human leukemia cells in combination with other inducers. Cancer Res 52: 4634-4641, 1992.

40. Masuda Y, Kawazoe N, Nakajo S, Yoshida T, Kuroiwa Y and Nakaya K: Bufalin induces apoptosis and influences the expression of apoptosis-related genes in human leukemia cells. Leuk Res 19: 549-556, 1995.

41. Yu CH, Kan SF, Pu HF, Jea Chien E and Wang PS: Apoptotic signaling in bufalin- and cinobufagin-treated androgen-dependent and -independent human prostate cancer cells. Cancer Sci 99: 2467-2476, 2008.

42. Zalatnai A and Molnár J: Review. Molecular background of chemoresistance in pancreatic cancer. In Vivo 21: 339-347, 2007.

43. Mimeault M, Hauke R and Batra SK: Recent advances on the molecular mechanisms involved in the drug resistance of cancer cells and novel targeting therapies. Clin Pharmacol Ther 83: 673-691, 2008. 